the fact that near the reciprocal lattice points the distribution may be taken as spherical.

Raman and Nath's more general formula

$$
d \sin \psi \sin (I-\varepsilon)=n \lambda \sin I
$$

is also only a purely geometrical relationship connecting the centre $C$ of a sphere with two points $O$ and $D$ on its surface and one point $P$ not on the sphere ( $P$ not necessarily in the plane of $C, O, D)$. Faxén's formula applies to the special case where $C, D, P$ are collinear.

Without detailed experimental information, the physical interpretation to be applied to such a general formula is largely a matter of choice. Part of the information required, of course, is precisely that

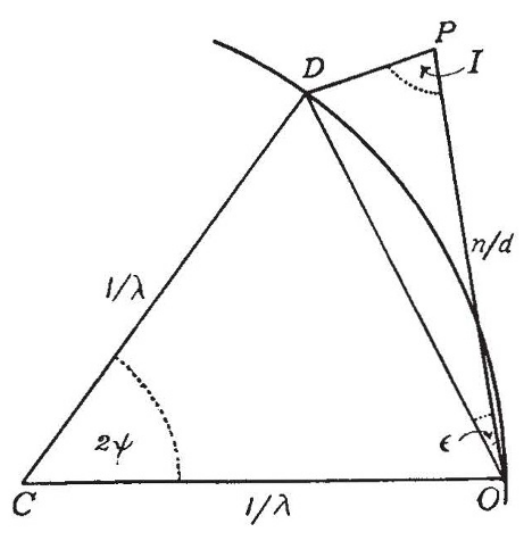

referred to by Preston, namely, the shape of the surfaces of isodiffusion (expression due to J. Laval), particularly when $\left(i-i_{B}\right)$ is not small. Such experimental observations, made in this Laboratory, show quite definitely that in general these surfaces are not spherical. For diamond, indeed, the reflecting region around the (111) reciprocal lattice point has been shown to possess six horns pointing along the six [100] directions ${ }^{3}$. Unfortunately, however, even this information is not enough. M. v. Laue ${ }^{4}$ has shown, for example, that for a finite crystal of octahedral shape, the reflecting region around a reciprocal lattice point has eight horns, but either wave theory will lead to similarly shaped regions provided that along certain crystal directions the atoms tend to remain always in phase.

The experimental information most to be desired at present is that relating the surfaces of isodiffusion for various reciprocal lattice points (to be experimentally determined) with other physical properties of the crystal, such as temperature, perfection, elasticity coefficients, and so on. Work along these lines is now in progress, but it is clear that the sub. ject opens up a very wide and fascinating field of research.

\section{K. Lonsdale.}

Davy Faraday Laboratory,

Albemarle Street,

London, W.1.

March 27.

Jauncey, NATURE 147, 146 (1941).

\& Preston, NATURE, 147, 358 (1941).

3 Lonsdale, K., and Smith, H., Proc. Roy. Soc., A, in the course publication.

‘v. Laue, Ann. Phys., 26, 55 (1936) ; 29, 211 (1937); Thomson and Cochrane, "Theory and Practice of Electron Diffraction" (Macmillan, 1939), p. 75 .

\section{A Property of Conjugated Systems}

IN a compound of the type $X .(\mathrm{CH}=\mathrm{CH})_{n} Y$, the conjugated system is an electronic conductor between the covalent groups $X$ and $Y$, and there must therefore be a correspondence between such chemical properties of the compound as leave the conjugated system intact and those of the covalent compound $X . Y$. This generalization has been recognized in the particular case of carbonyl compounds, but its wide value as a very general means of insight into the reactivity, as well as the mechanism of reactivity, of the compound $X . Y$, seems to have been overlooked.

The most readily available conjugated systems are those of ortho- and para-benzene derivatives, and the generalization at once disposes of the old supposition of a fundamental contrast between aromatic and open-chain chemistry. Nevertheless, the possibility of nitrating paraffins which, in this light, follows from that of nitrating alkyl benzenes, has only latterly been realized and put into technical operation. This example also serves to illustrate the efficacy of the aromatic resonance in promoting reactivity, a factor which must be allowed for in applying the principle. Although it is for this reason safer to deduce the behaviour of $X Y$ from that of the conjugated compound than to do the reverse, nevertheless it is clear that the possibility of chlorinating or nitrating $X \mathrm{H}$ to $X \mathrm{Cl}$ or $X \mathrm{NO}_{2}$ can provide a criterion of the ortho-para directive effect in aromatic substitution.

Again, the mechanism of para-nitrosation of dimethyl aniline must be reproduced in that of dimethylamine, and it is obviously not permissible to regard the latter as involving the salt of the base if, as is accepted, the former involves the free base. The widely accepted view, based on the results of kinetic measurements ${ }^{2}$, that nitrosation and diazotization involve the salts of bases, is therefore untenable; the function of the protons, which such measurements show to participate in the reaction, must therefore be to activate the nitrous acid. It is, however, hoped to show in a fuller discussion of this reaction that other reasons independently point to the same conclusion.

The principle also constitutes a bridge between organic and inorganic chemistry, of the more value because in the latter the study of reactivity and mechanism is little advanced. Thus the mode of oxidation of hydrogen peroxide, hydroxylamine, and hydrazine respectively must correspond to those of hydroquinone, $p$-aminophenol and $p$-phenylene diamine. The $o-p$ nitration of chlorobenzene must be reflected in that of hydrogen chloride, which doubtless represents the first stage in the familiar formation of nitrosyl chloride and chlorine. The nitration of aniline (as acetanilide) shows that, as indeed Franchimont ${ }^{2}$ surmised, nitramide is an intermediate in the preparation of nitrous oxide from ammonium nitrate; similarly, the formation of nitrogen from ammonium nitrite is, as has also been shown kinetically ${ }^{1}$, a case of diazotization.

College of Technology, Manchester, 1. March 28 .

1 Hantzsch and Schumann, Ber., 321, 1691 (1899); Taylor, J. Ohem. Soc. 1099,1897 (1928); 2032 (1929); Schmid, Z. Elektrochem., $39,863(1933) ; 42,579(1936)$

Rec. Trav. Chim., 2, 94 (1883) 